\title{
Effect of Teneligliptin versus Sulfonylurea on Major Adverse Cardiovascular Outcomes in People with Type 2 Diabetes Mellitus: A Real-World Study in Korea
}

\author{
Da Hea Seo ${ }^{1, *}$, Kyoung Hwa $\mathrm{Ha}^{2}$,*, So Hun $\mathrm{Kim}^{1}$, Dae Jung Kim²
}

${ }^{1}$ Division of Endocrinology and Metabolism, Department of Internal Medicine, Inha University School of Medicine, Incheon; ${ }^{2}$ Department of Endocrinology and Metabolism, Ajou University School of Medicine, Suwon, Korea

Background: Results regarding the cardiovascular (CV) effects of dipeptidyl peptidase-4 (DPP-4) inhibitors are inconsistent. This study aimed to assess the effects of teneligliptin, a DPP-4 inhibitor, on the risk of major CV outcomes in type 2 diabetes mellitus (T2DM) patients compared to sulfonylurea.

Methods: From January 1, 2015 to December 31, 2017, we conducted a retrospective cohort study using the Korean National Health Insurance Service database. A total of 6,682 T2DM patients who were newly prescribed DPP-4 inhibitors or sulfonylurea were selected and matched in a 1:1 ratio by propensity score. The hazard ratios (HRs) for all-cause mortality, hospitalization for heart failure (HHF), all-cause mortality or HHF, myocardial infarction (MI), stroke, and hypoglycemia were assessed.

Results: During 641 days of follow-up, the use of teneligliptin was not associated with an increased risk of all-cause mortality (HR, 1.00; $95 \%$ confidence interval [CI], 0.85 to 1.19), HHF (HR, 0.99; 95\% CI, 0.86 to 1.14), all-cause mortality or HHF (HR, 1.02; $95 \%$ CI, 0.90 to 1.14), MI (HR, 0.90; 95\% CI, 0.68 to 1.20), and stroke (HR, 1.00; 95\% CI, 0.86 to 1.17) compared to the use of sulfonylurea. However, it was associated with a significantly lower risk of hypoglycemia (HR, $0.68 ; 95 \% \mathrm{CI}, 0.49$ to 0.94 ) compared to sulfonylurea therapy.

Conclusion: Among T2DM patients, teneligliptin therapy was not associated with an increased risk of CV events including HHF, but was associated with a lower risk of hypoglycemia compared to sulfonylurea therapy.

Keywords: Hypoglycemic agents; Cardiovascular diseases; Dipeptidyl-peptidase IV inhibitors; Heart failure; Hypoglycemia

Received: 17 July 2020, Revised: 19 November 2020,

Accepted: 25 November 2020

Corresponding authors: So Hun Kim

Division of Endocrinology and Metabolism, Department of Internal Medicine, Inha University School of Medicine, 27 Inhang-ro, Jung-gu, Incheon 22332, Korea

Tel: +82-32-890-3573, Fax: +82-32-882-6578, E-mail: shoney@inha.ac.kr

Dae Jung Kim

Department of Endocrinology and Metabolism, Ajou University School of Medicine, 164 World cup-ro, Yeongtong-gu, Suwon 16499, Korea

Tel: +82-31-219-5128, Fax: +82-31-219-4497, E-mail: djkim@ajou.ac.kr
Copyright $\odot 2021$ Korean Endocrine Society

This is an Open Access article distributed under the terms of the Creative Commons Attribution Non-Commercial License (https://creativecommons.org/ licenses/by-nc/4.0/) which permits unrestricted non-commercial use, distribution, and reproduction in any medium, provided the original work is properly cited.

*These authors contributed equally to this work. 


\section{INTRODUCTION}

Dipeptidyl peptidase 4 (DPP-4) inhibitors are incretin-based antidiabetic drugs that are widely prescribed for type 2 diabetes mellitus (T2DM) patients owing to their ease in use, fair glycemic control, neutral effect on body weight, and low occurrences of hypoglycemic episodes [1]. However, there have been concerns about their possible unfavorable effects on cardiovascular (CV) outcomes as cardiovascular outcome trials (CVOTs) with different DPP-4 inhibitors having shown inconsistent results [2].

To date, several CVOTs with four DPP-4 inhibitors have been published, including the Saxagliptin Assessment of Vascular Outcomes Recorded in Patients with Diabetes Mellitus-Thrombolysis in Myocardial Infarction (SAVOR-TIMI 53) trial, the EXamination of cArdiovascular outcoMes with alogliptIN (EXAMINE trial), the Trial Evaluating Cardiovascular Outcomes with Sitagliptin (TECOS), the Cardiovascular and Renal Microvascular Outcome Study With Linagliptin (CARMELINA), and the Cardiovascular Outcome Study of Linagliptin Versus Glimepiride in Patients With Type 2 Diabetes (CAROLINA), which have all shown the CV safety of DPP-4 inhibitors compared to placebo or sulfonylurea [3-7]. However, an increase in the rate of hospitalization for heart failure (HHF) was reported with saxagliptin, and an analysis of the EXAMINE trial data has also shown a nonsignificant increase in HHF with alogliptin use $[5,8]$. In a recent meta-analysis with randomized controlled trials and observational studies, the use of DPP-4 inhibitors was not associated with increased risk of $\mathrm{CV}$ outcomes, including $\mathrm{HHF}$, compared to the control group [9]. However, in a recent observational study with a nationwide database, the use of DPP4 inhibitors as an add-on therapy to metformin was associated with a higher risk of HHF compared to sulfonylurea [10], while other studies have demonstrated that the use of DPP-4 inhibitors was associated with a lower risk of cardiovascular disease (CVD) events, including HHF, compared to the use of sulfonylurea $[11,12]$.

Despite numerous CV outcome trials and observational studies, the relative effect of DPP-4 inhibitors on CV outcomes in T2DM patients remains uncertain [9-14]. Additionally, there is a possibility that DPP-4 inhibitors exhibit within-class differences in CV outcomes [15] since there are differences in their duration of action, metabolism, elimination, and compoundspecific characteristics [16]. Teneligliptin, a new addition to the DPP-4 inhibitor class, was approved for the treatment of T2DM in Japan in 2012 and subsequently in Korea in 2014. Many clinical trials have assessed its safety and efficacy [17]; however, no
CVOTs have assessed its CV safety. Therefore, this observational study aimed to investigate its effect on a broad range of $\mathrm{CV}$ outcomes in a real-world setting using the Korean National Health Insurance Service (NHIS) database.

\section{METHODS}

\section{Data sources}

From January 2015 to December 2017, patient data were obtained from the Korean NHIS database, which covers $98 \%$ of the population $[18,19]$. A broad spectrum of variables are included in this database, which includes sex, socioeconomic status, medical care history (medical treatment and health examinations), medical care institutions, diagnosis code, surgery code, and data on drug prescription name, dose of drugs, and date of prescription.

\section{Study subjects}

In this retrospective study, we selected patients who were at least 20 years old, had at least 1 recorded diagnosis of T2DM (International Classification of Diseases [ICD]-10 codes E1114), and were initiated glucose lowering drugs, including metformin, sulfonylurea, DPP-4 inhibitors, sodium-glucose cotransporter-2 (SGLT2) inhibitor, thiazolidinedione (TZD), glucagon-like peptide-1 receptor agonist, alpha-glucosidase inhibitor (AGI), and insulin. New users were defined as those whose date of initial exposure (index date) to DPP-4 inhibitors and other glucose lowering drugs was during the study period (January 1, 2015 and December 31, 2017) and who had no prior exposure in the previous year. Type 1 diabetes (ICD-10 codes E10) or gestational diabetes mellitus (ICD-10 codes O24) patients were excluded from the study. Among these patients, we identified those who had been newly prescribed with teneligliptin or sulfonylurea (glimepiride, glipizide, glibenclamide, or gliclazide) (Supplemental Fig. S1). Subjects were followed until the first occurrence of any following: (1) outcomes of interest, (2) death, or (3) end of the study period (December 31, 2017). This study protocol was approved by the Institutional Review Board of Ajou University Hospital, Suwon, Korea (No. AJIRBMED-EXP-18-297) and the requirement for informed consent was waived by the board.

\section{Outcomes}

The outcome of interest for this study was defined as any hospitalization or visit to an emergency department because of CVD with a primary diagnosis of HHF (ICD-10 codes I50), myocar- 
dial infarction (MI) (ICD-10 codes I21, I22), and stroke (ICD10 codes I60-64). Modified three-point major adverse cardiovascular event (modified 3P-MACE) was defined as the composite of MI, stroke, or all-cause mortality. All-cause mortality was defined by death status in the NHIS database, which was linked to the National Death Registry using unique resident registration numbers. Severe hypoglycemia was defined as hospitalization for any of the following diagnosis of hypoglycemia (ICD-10 codes E10.0, E11.0, E11.63, E12.0, E13.0, E14.0, and E16.0-2).

\section{Covariates}

Baseline characteristics within 1 year prior to the index date were used for analysis. We considered following as confounding variables: age, sex, index year, use of other glucose lowering drugs, use of CVD risk treatment drugs, chronic kidney disease (CKD), diabetic macrovascular or microvascular complications, previous history of severe hypoglycemia, cancer, and frailty. A list of variables with the corresponding ICD-10 codes, procedures codes and treatment codes is provided in Supplemental Table S1.

\section{Statistical analysis}

Categorical variables are described by frequencies and percentages, and continuous variables are described as mean \pm standard deviation. Propensity scores were matched in a 1:1 ratio using a caliper width that was equal to 0.25 of the standard deviation of the logits [20]. Potential risk factors related to outcome and confounding variables associated with both treatment status (teneligliptin or sulfonylurea) and outcome were included as propensity score covariates as follows: age, sex, index year, use of other glucose lowering drugs, use of CVD risk treatment drugs, CKD, diabetic macrovascular or microvascular complications, previous history of severe hypoglycemia, cancer, and frailty. Before and after propensity score matching, the covariate balance was calculated as standardized differences; significant imbalances were defined as standardized differences of $\geq 10 \%$ [21]. Only the first occurrence of each outcome was included and the crude incidence rate (IR) in each group was calculated as the number of incident events divided by the total number of 100 personyears at risk. Time to first event for the teneligliptin and sulfonylurea groups was assessed using Kaplan-Maier plots and the log-rank test. Cox proportional hazards models were used to calculate the hazard ratios (HR) and 95\% confidence intervals (CI) for each outcome. The primary analysis used an intentionto-treat approach, in which all individuals were followed from the index date until the outcome of interest, death, or end of the study period, whichever came first. Analyses for all outcomes were then repeated across multiple patient subgroups to examine whether the associations of teneligliptin and sulfonylurea with $\mathrm{CV}$ outcomes differed based on patient demographics or clinical and treatment characteristics. These pre-specified subgroup analyses were compared for multiple covariates including age, sex, use of statin or insulin, and history of CKD, CVD, and cancers. To test the stability of the findings, a sensitivity analysis using an on-treatment approach was performed, where follow-up was censored at discontinuation of the index treatment, outcome of interest, or death, whichever came first. All analyses were performed using SAS software version 9.4 (SAS Institute Inc., Cary, NC, USA) and survival curves were generated using "survival" R package version 2.42-6 (R Foundation for Statistical Computing, Vienna, Austria).

\section{RESULTS}

\section{Study population}

The study included a total of 27,122 patients: 3,341 patients in the teneligliptin group and 23,781 patients in the sulfonylurea group (Supplemental Fig. S1). Prior to propensity matching, patients initiated on teneligliptin had higher rates of diabetic nephropathy, similar rates of $\mathrm{CV}$ comorbidities, and lower rates of frailty. The use of statin and angiotensin receptor blockers (ARB) was greater in the teneligliptin group while the use of aspirin was similar. Patients in both groups had similar rates of other glucose-lowering drugs except AGI, the use of which was higher in the sulfonylurea group (Supplemental Table S2).

Following propensity matching, the cohort included 6,682 patients: 3,341 patients in the teneligliptin group and 3,341 patients in the sulfonylurea group (Table 1). Baseline characteristics were well balanced between groups. The mean age of the subjects was 62 years and $41 \%$ of the subjects were women. The most commonly used glucose-lowering drugs in combination with teneligliptin or sulfonylurea were metformin $(62 \%)$, insulin (36\%), TZD (6.6\%), and SGLT2 inhibitors (3.2\%). Those who were prescribed low-dose aspirin, statins, angiotensin converting enzyme inhibitors, and ARB were $27 \%, 52 \%$, $3.5 \%$, and $45.6 \%$, respectively. Of the total participants, $35 \%$ had a history of CVD, $7 \%$ had CKD, and $20 \%$ had a history of cancer.

\section{Cardiovascular outcomes}

The median follow-up duration was 641 days (interquartile 
Table 1. Baseline Characteristics after Propensity Score Matching $(n=3,341$ pairs $)$

\begin{tabular}{lrrc}
\hline Characteristic & Teneligliptin & Sulfonylurea & $\begin{array}{c}\text { Standardized } \\
\text { difference, } \%\end{array}$ \\
\hline Age, yr & $62.8 \pm 13.7$ & $62.4 \pm 14.0$ & 2.9 \\
Women & $1,389(41.6)$ & $1,338(40.0)$ & 3.1 \\
Index year & & & \\
2015 & $420(12.6)$ & $432(12.9)$ & 1.1 \\
2016 & $1,512(45.3)$ & $1,535(45.9)$ & 1.4 \\
2017 & $1,409(42.2)$ & $1,374(41.1)$ & 2.1
\end{tabular}

Glucose-lowering drugs

$\begin{array}{lccc}\text { Metformin } & 2,055(61.5) & 2,086(62.4) & 1.9 \\ \text { SU } & 0 & 0 & 0.0 \\ \text { DPP-4i } & 0 & 0 & 0.0 \\ \text { SGLT2i } & 108(3.2) & 121(3.6) & 2.1 \\ \text { TZD } & 219(6.6) & 208(6.2) & 1.3 \\ \text { GLP-1RA } & 1(0.0) & 0 & 2.4 \\ \text { Meglitinide } & 73(2.2) & 83(2.5) & 2.0 \\ \text { AGI } & 76(2.3) & 65(1.9) & 2.3 \\ \text { Insulin } & 1,217(36.4) & 1,184(35.4) & 2.1\end{array}$

CVD risk treatment

\begin{tabular}{lccc} 
Low-dose acetylsalicylic & $909(27.2)$ & $891(26.7)$ & 1.2 \\
acid & & & \\
Statin & $1,768(52.9)$ & $1,722(51.5)$ & 2.8 \\
Antihypertensive therapy & $2,090(62.6)$ & $2,077(62.2)$ & 0.8 \\
ACE inhibitors & $117(3.5)$ & $115(3.4)$ & 0.3 \\
ARB & $1,525(45.6)$ & $1,460(43.7)$ & 3.9 \\
Dihydropyridines & $1,009(30.2)$ & $975(29.2)$ & 2.2 \\
Low-ceiling diuretics & $325(9.7)$ & $321(9.6)$ & 0.4 \\
Beta blockers & $847(25.4)$ & $802(24.0)$ & 3.1 \\
Non-hydropyridines & $146(4.4)$ & $134(4.0)$ & 1.8 \\
High-ceiling diuretics & $699(20.9)$ & $645(19.3)$ & 4.0 \\
Aldosterone antagonists & $236(7.1)$ & $222(6.6)$ & 1.7 \\
Warfarin & $86(2.6)$ & $83(2.5)$ & 0.6 \\
CVD & $1,196(35.8)$ & $1,159(34.7)$ & 2.3 \\
Myocardial infarction & $137(4.1)$ & $122(3.7)$ & 2.3 \\
CABG & $8(0.2)$ & $7(0.2)$ & 0.6 \\
PCI with stent & $65(1.9)$ & $72(2.2)$ & 1.5 \\
Unstable angina & $193(5.8)$ & $202(6.0)$ & 1.1 \\
Angina pectoris & $645(19.3)$ & $643(19.2)$ & 0.2 \\
Heart failure & $447(13.4)$ & $454(13.6)$ & 0.6 \\
Atrial fibrillation & $187(5.6)$ & $185(5.5)$ & 0.3 \\
\hline & & $($ Continued to the next $)$ \\
\hline
\end{tabular}

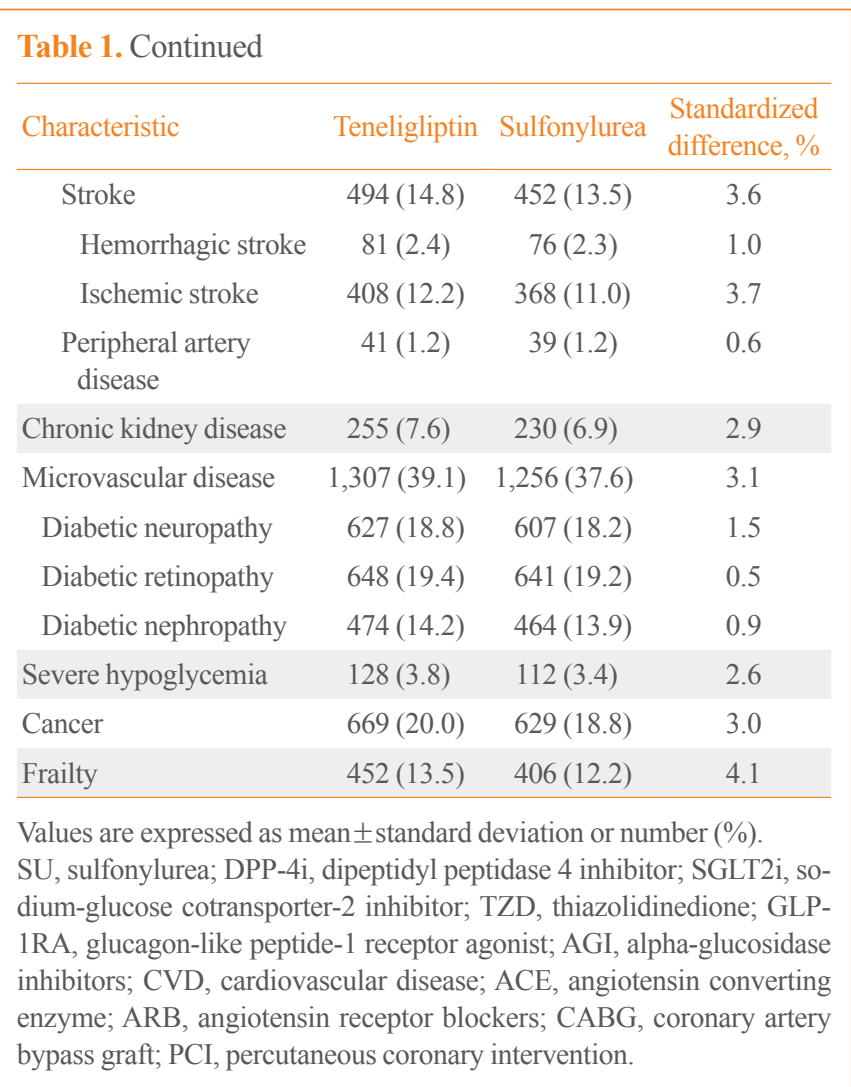

range, 494 to 872 ). The IRs per 100 person-years for the allcause mortality were 7.02 in the teneligliptin group and 6.96 in the sulfonylurea group (Table 2). Initiation of teneligliptin treatment was not associated with an increased risk of all-cause mortality compared to sulfonylurea treatment (HR, 1.00; 95\% CI, 0.85 to 1.19 ) (Table 2, Fig. 1A). The IRs per 100 person-years for HHF were 10.14 in the teneligliptin group and 10.12 in the sulfonylurea group (Table 2). The initiation of teneligliptin was not associated with an increased risk of HHF compared to sulfonylurea (HR, 0.99; 95\% CI, 0.86 to 1.14) (Table 2, Fig. 1B). Overall, initiation of teneligliptin versus sulfonylurea was not associated with increased risk of all-cause mortality or HHF (HR, 1.02; 95\% CI, 0.90 to 1.14) (Table 2, Fig. 1C).

With regards to MI, the IRs per 100 person-years were 2.47 in the teneligliptin group and 2.70 in the sulfonylurea group. The HR of MI in teneligliptin users was 0.90 (95\% CI, 0.68 to 1.20$)$ (Table 2, Fig. 1D). The IRs per 100 person-years for stroke were 9.31 in the teneligliptin group and 9.09 in the sulfonylurea group (Table 2). The initiation of teneligliptin was not associated with an increased risk of stroke compared to sulfonylurea (HR, 1.00; 95\% CI, 0.86 to 1.17) (Table 2, Fig.1E). Overall, the IRs per 100 person-years for the modified 3P-MACE were 17.01 in the teneligliptin and 17.42 in the sulfonylurea groups 
Table 2. Event Rates and HRs for the Cardiovascular Outcomes, and Severe Hypoglycemia (Intent-to-Treat Analysis)

\begin{tabular}{llrrr}
\hline \multirow{2}{*}{ Variable } & \multicolumn{2}{c}{ Event rate/100 patient-years } & HR $(95 \%$ CI $)$ & P value \\
\cline { 2 - 3 } ACM & Teneligliptin & Sulfonylurea & & \\
\hline HHF & 7.02 & 6.96 & $1.00(0.85-1.19)$ & 0.966 \\
ACM+HHF & 10.14 & 10.12 & $0.99(0.86-1.14)$ & 0.897 \\
Myocardial infarction & 15.73 & 15.30 & $1.02(0.90-1.14)$ & 0.772 \\
Stroke & 2.47 & 2.70 & $0.90(0.68-1.20)$ & 0.484 \\
Myocardial infarction, stroke or all-cause mortality (modified 3P-MACE) & 17.01 & 9.09 & $1.00(0.86-1.17)$ & 0.961 \\
Severe hypoglycemia & 1.65 & 17.42 & $0.96(0.86-1.08)$ & 0.515 \\
\hline
\end{tabular}

All outcomes were assessed using a Cox proportional hazards model for comparing teneligliptin with sulfonylurea (reference) after propensity matching. HR, hazard ratio; CI, confidence interval; ACM, all-cause mortality; HHF, hospitalization for heart failure; 3P-MACE, 3-point major adverse cardiovascular event.

(Table 2). Initiation of teneligliptin treatment was not associated with an increased risk of the modified 3P-MACE compared to sulfonylurea treatment (HR, 0.96; 95\% CI, 0.86 to 1.08) (Table 2, Fig. 1F). In the sensitivity analyses using an on-treatment approach, the association between teneligliptin initiation and all CV outcomes remained consistent (Table 3 ).

In all subgroup analyses, for all $\mathrm{CV}$ outcomes, no meaningful interactions were observed with the patients' demographic, clinical, or treatment characteristics, suggesting that none of these variables had an effect on the association between teneligliptin and CVD outcomes (Supplemental Figs. S2-7). Importantly, in patients with and without established CVD at baseline, the use of teneligliptin was not associated with increased risks of allcause mortality, HHF, all-cause mortality or HHF, MI, stroke, and modified 3P-MACE (Fig. 2).

\section{Outcome of severe hypoglycemia}

For severe hypoglycemia, the incidence per 100 person-years was 1.65 in the teneligliptin and 2.39 in sulfonylurea groups. The risk was significantly lower in the teneligliptin group compared to that in the sulfonylurea group (HR, 0.68; 95\% CI, 0.49 to 0.94 ) (Table 2, Fig. $1 \mathrm{G}$ ). In a sensitivity analysis, the magnitude of the association between teneligliptin treatment and lower risk of hypoglycemia was further increased, strongly favoring teneligliptin compared to sulfonylurea (HR, 0.40; 95\% CI, 0.24 to 0.68 ) (Table 3 ). In subgroup analyses for hypoglycemia, no meaningful interactions were observed (Fig. 3).

\section{DISCUSSION}

In this population-based retrospective study, we found that teneligliptin was not associated with an increased risk of mortality and CV outcomes including HHF, compared to sulfonylurea, in T2DM patients. It is also worth noting that the results were consistent across various patient subgroups including those with and without established CVD. Moreover, this study showed that the use of teneligliptin was associated with a lower risk of hypoglycemic events compared to sulfonylurea. The results of this study are in accordance with those of a recent randomized clinical trial comparing linagliptin and glimepiride, which demonstrated a non-inferior risk of CV outcomes [6].

Beyond their effective glucose-lowering effect, there is accumulating evidence that DPP-4 inhibitors exert cardio-protective effects by exerting neutral to modest beneficial effects on body weight, blood pressure, lipid profile, and inflammatory markers, thus attenuating oxidative stress and improving endothelial dysfunction in animal models [22,23]. However, these positive cardiac effects are yet to be clinically confirmed. Moreover, randomized controlled CV outcome trials with different DPP-4 inhibitors have shown inconsistent results particularly for HHF. In the SAVOR-TIMI study, saxagliptin use did not increase CV event risk; however, HHF incidence was higher in saxagliptintreated patients compared with those in the placebo group (HR, 1.27 ; $95 \%$ CI, 1.07 to $1.51 ; P=0.007$ ) [4]. The subsequent trial with alogliptin in diabetic patients who were recently hospitalized for acute coronary syndrome showed a neutral effect on $\mathrm{CV}$ events; however, a non-significant trend of a higher rate of HHF was observed in the alogliptin group compared to the placebo group [8]. The most recent trials, TECOS and CARMELINA, demonstrated that both sitagliptin and linagliptin were not associated with an increased risk of CVD including HHF [7]. However, no trials have elucidated the relationship between 

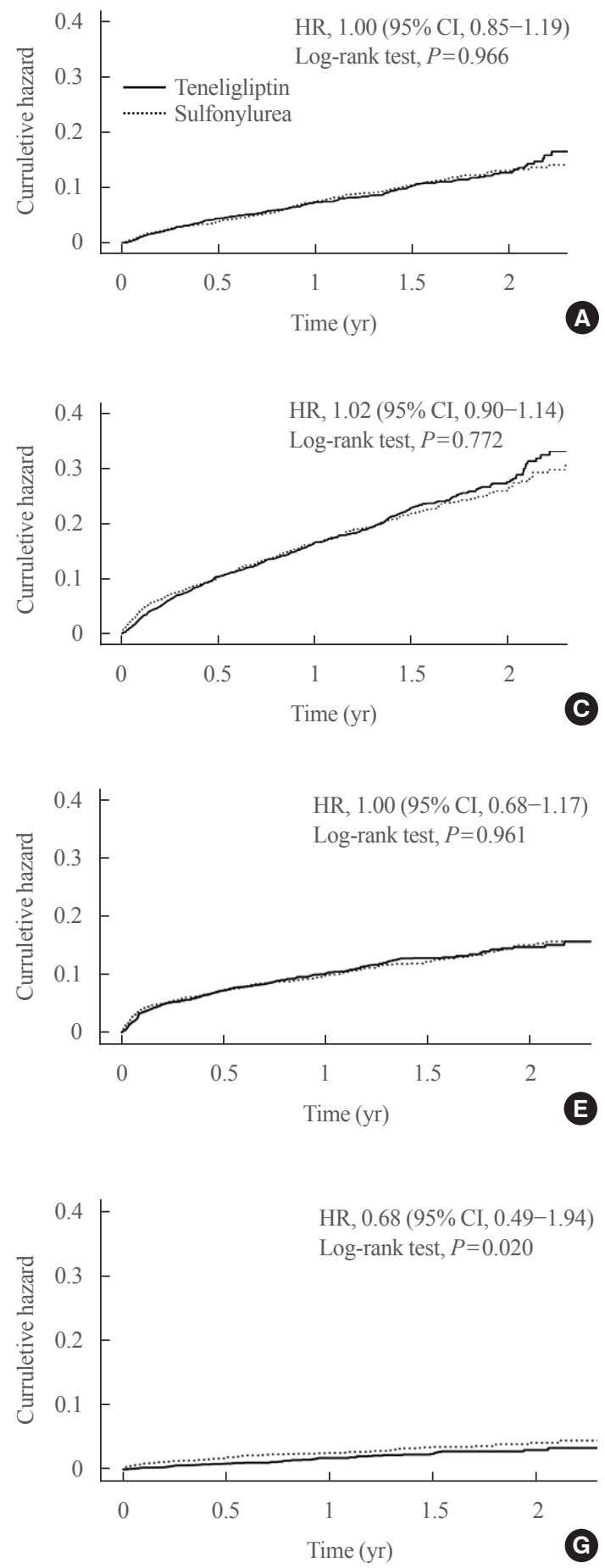

teneligliptin and CV outcomes. Using the Korean NHIS database, the present retrospective population-based study demonstrated the CV safety of teneligliptin compared to sulfonylurea, further elucidating the safety of teneligliptin.

In a recent observational study with a large nationwide diabetic cohort of 113,051 patients with T2DM, DPP-4 inhibitors
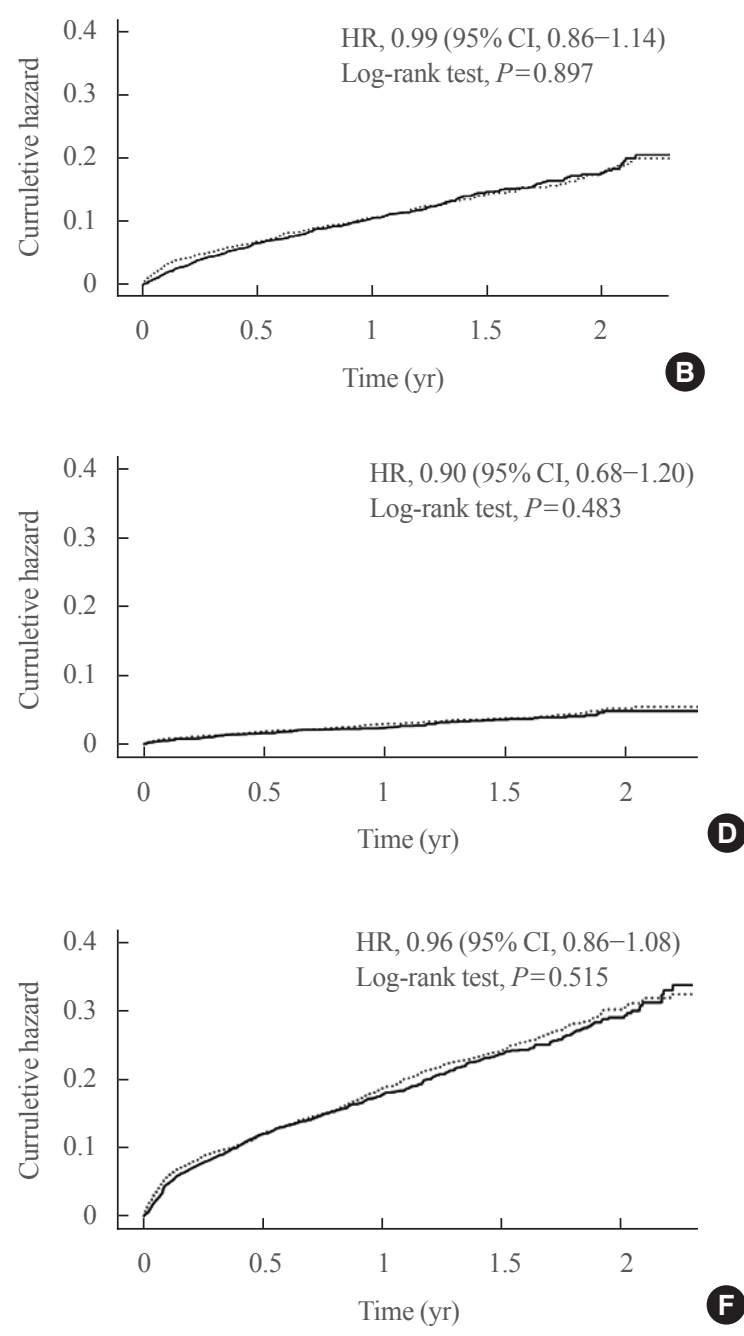

Fig. 1. Comparison of the cumulative incidence for all cardiovascular outcomes and severe hypoglycemia. Comparison of the cumulative incidence for all-cause mortality (A), hospitalization for heart failure (B), hospitalization for heart failure or all-cause mortality (C), myocardial infarction (D), stroke (E), modified 3-point major adverse cardiovascular event outcomes (myocardial infarction, stroke, or all-cause mortality) (F), and severe hypoglycemia (G). $\mathrm{HR}$, hazard ratio; $\mathrm{CI}$, confidence interval.

as a second-line add-on treatment showed a lower risk of primary composite $\mathrm{CV}$ outcomes including HHF, compared to sulfonylurea (HR, 0.85 ; $95 \%$ CI, 0.74 to 0.98 ) [24]. Another population-based cohort study in the UK demonstrated that the DPP-4 inhibitor-metformin combination was associated with a lower risk of major $\mathrm{CV}$ events and all-cause mortality compared to the 
Table 3. Event Rates and HRs for the Cardiovascular Outcomes, and Severe Hypoglycemia (on Treatment Analysis)

\begin{tabular}{|c|c|c|c|c|}
\hline \multirow{2}{*}{ Variable } & \multicolumn{2}{|c|}{ Event rate/100 patient-years } & \multirow{2}{*}{$\mathrm{HR}(95 \% \mathrm{CI})$} & \multirow{2}{*}{$P$ value } \\
\hline & Teneligliptin & Sulfonylurea & & \\
\hline $\mathrm{ACM}$ & 3.66 & 2.83 & $1.30(0.86-1.96)$ & 0.213 \\
\hline HHF & 12.28 & 14.45 & $0.86(0.70-1.05)$ & 0.147 \\
\hline $\mathrm{ACM}+\mathrm{HHF}$ & 15.26 & 16.69 & $0.93(0.77-1.11)$ & 0.415 \\
\hline Myocardial infarction & 2.98 & 3.98 & $0.76(0.51-1.23)$ & 0.175 \\
\hline Stroke & 12.59 & 14.34 & $0.90(0.74-1.10)$ & 0.311 \\
\hline Myocardial infarction, stroke or all-cause mortality (modified 3P-MACE) & 18.33 & 20.16 & $0.93(0.78-1.10)$ & 0.392 \\
\hline Severe hypoglycemia & 1.29 & 3.31 & $0.40(0.24-0.68)$ & 0.001 \\
\hline
\end{tabular}

All outcomes were assessed using a Cox proportional hazards model for comparing teneligliptin with sulfonylurea (reference) after propensity matching. $\mathrm{HR}$, hazard ratio; CI, confidence interval; ACM, all-cause mortality; HHF, hospitalization for heart failure; 3P-MACE, 3-point major adverse cardiovascular event.

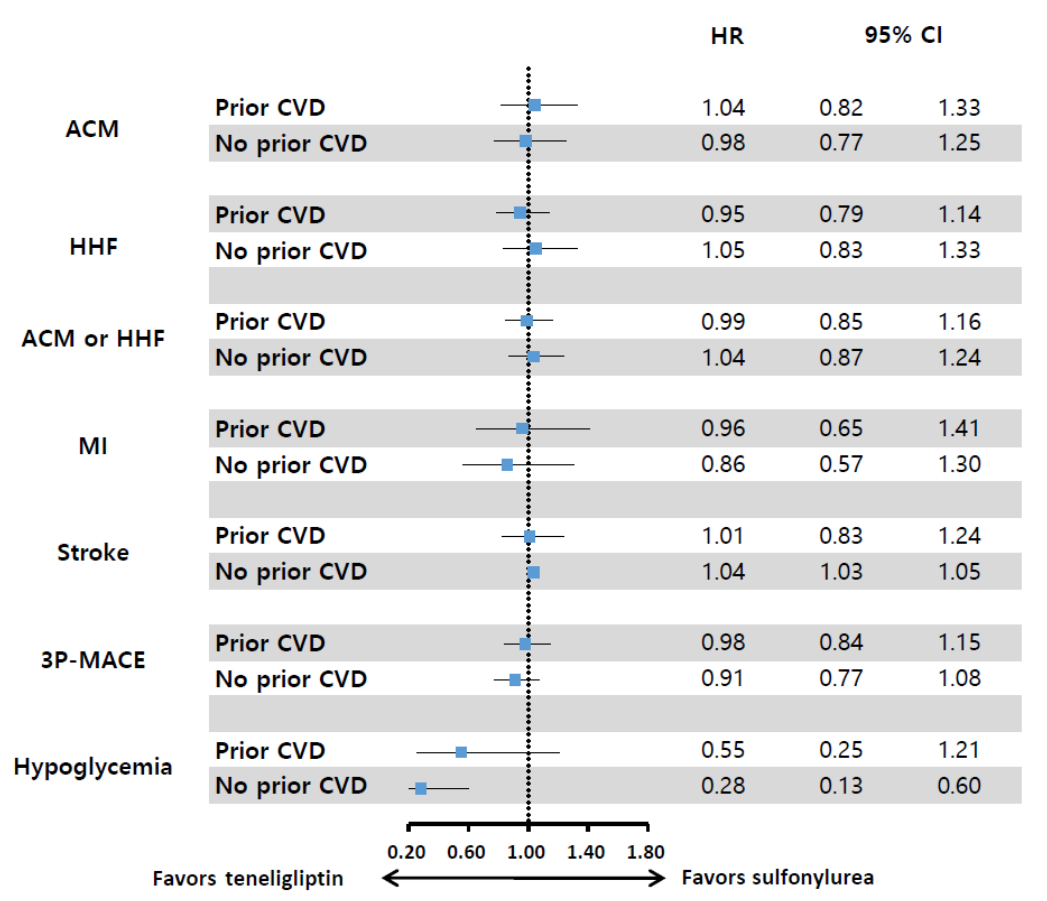

Fig. 2. Cardiovascular outcomes in subgroups of patients with and without established cardiovascular disease at baseline for all outcomes. $\mathrm{HR}$, hazard ratio; CI, confidence interval; ACM, all-cause mortality; CVD, cardiovascular disease; HHF, hospitalization for heart failure; MI, myocardial infarction; 3P-MACE, modified 3-point major adverse cardiovascular events outcomes (myocardial infarction, stroke, or all-cause mortality).

sulfonylurea and metformin combination (HR, 0.62; 95\% CI, 0.40 to 0.98 ) [25]. On the other hand, in a retrospective cohort study with T2DM comprising patients with no previous history of congestive heart failure (CHF), using the Cleveland Clinic enterprise-wide electronic health record system, it was determined that the use of a combination of a DPP-4 inhibitor and metformin was not associated with a lower risk of CV events, while the risk of CHF was higher (HR, 1.104; 95\% CI, 1.04 to
1.17) compared to the sulfonylurea and metformin groups [26].

The results of recent observational studies of DPP-4 inhibitors versus sulfonylurea on $\mathrm{CV}$ risk using real world data from the Korean NHIS or Korean Health Insurance Review and Assessment Service were conflicting. In a population study by Chin et al. [14], the use of a DPP-4 inhibitor was not associated with an increased CVD risk compared to the use of glimepiride, regardless of a baseline CVD history. Additionally, DPP-4 in- 


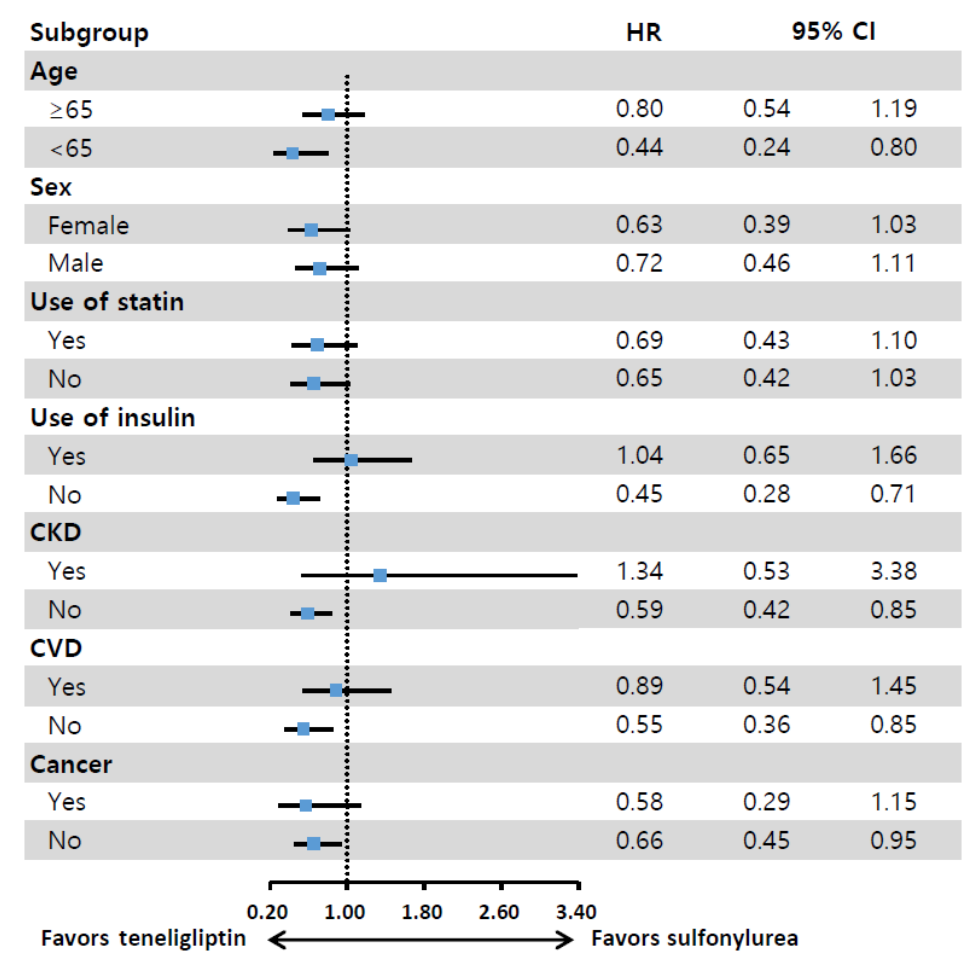

Fig. 3. Subgroup analyses for hypoglycemia by age, gender, statin and insulin use, chronic kidney disease, and cardiovascular disease and cancer at baseline. HR, hazard ratio; CI, confidence interval; CKD, chronic kidney disease; CVD, cardiovascular disease.

hibitor therapy demonstrated protective effects against heart failure compared to sulfonylurea [14]. Similarly, both Lee et al. [12] and Kim et al. [13] reported that DPP-4 inhibitor treatment alone or as an add-on therapy to metformin lowered HHF risk compared to the use of sulfonylureas, regardless of a history of CVD. On the other hand, two Korean population studies reported that DPP-4 inhibitor therapy did not increase atherosclerotic CVD risk, with the exception of HHF, compared to glimepiride use $[10,11]$. There was an increased risk of HHF in patients on DPP-4 therapy, especially those without baseline CVD, and was consistent with post-hoc analysis of the EXAMINE [8] and SAVOR-TIMI trials [27]. However, teneligliptin users were not included in any of the previous studies. It is assuring that in the present study with teneligliptin, HHF risk was not increased compared to sulfonylurea, regardless of a baseline CVD history. Our finding is further supported by a small study in patients with T2DM and asymptomatic left ventricular (LV) dysfunction, where teneligliptin treatment improved LV diastolic and systolic function, even at an early stage [28].

Despite numerous randomized clinical trials, observational studies, and meta-analyses of randomized clinical trials, it remains unclear whether the $\mathrm{CV}$ effect of DPP-4 inhibitors varies.
It is worth noting that each study had different patient populations with different CV risks. Moreover, DPP-4 is a ubiquitous protease that degrades several substances including endogenous incretin hormones. Additionally, DPP-4 inhibition may present unknown and unexpected deleterious effects resulting from offtarget effects. Nevertheless, our results suggested that a DPP-4 inhibitor such as teneligliptin may be a safe option for add-on therapy owing to its neutral effect on CV risk and low risk of hypoglycemia when additional glycemic control is needed.

The IRs of all-cause mortality and HHF were higher in this study compared to other real world observation studies [29,30]. However, as the study populations included in those studies $[29,30]$ had quite different baseline characteristics compared to our study population, this may have led to the difference in rates of mortality and HHF. The mean age of study subjects in our study was approximately 10 years older than other studies $[29,30]$ (63 years vs. 53-56 years) and we included a higher proportion of patients with underlying medical conditions including CVD, CHF, and CKD, which are associated with higher risk for all-cause mortality and HHF. Previous studies have indicated that older people with diabetes are at an excessively higher risk for vascular complications due to their potentially 
longer disease duration [31]. Data from the National Diabetes Surveillance System indicates that the overall absolute risk for CVD increases dramatically with age, and age strongly predicts diabetes-related CHF, ischemic heart disease (IHD), and stroke [32]. In a prospective population-based cohort study, there was a sequential increase in IRs of heart failure for all patients in 5-year age categories from 55-59 to $\geq 90$ years of age [33]. Similarly, in an epidemiological study with T2DM patients, the odds ratio for IHD, stroke, heart failure and MI all increased sequentially with each increase in 5-year age category as compared with the age group 35 to 39 used as a reference [34].

DPP-4 inhibitors have consistently been attributed to a lower risk of hypoglycemia, which has been well highlighted in multiple treatment guidelines for diabetes $[35,36]$. Our study with teneligliptin has demonstrated that it is associated with a $60 \%$ lower risk of hypoglycemia compared to sulfonylurea treatment. Moreover, previous placebo-controlled trials have shown that there was no difference in the risk of hypoglycemia between the DPP-4 inhibitor and placebo groups [36-39]. This is particularly important for frail, elderly patients who are at higher risk of hypoglycemia, which can lead to significant morbidity and mortality [40]. DPP-4 inhibitors have been safely used in older populations [41]. Moreover, these drugs, especially teneligliptin, can be prescribed for patients with moderate to severe CKD and end stage renal disease, without dose adjustment [42].

There are several limitations in this study. First, there is a possibility of residual, unmeasured, and uncontrolled confounding factors, due to the observational nature of the study, using a health insurance claim database. However, we used robust statistical techniques such as propensity matching (age, sex, index year, use of other glucose-lowering drugs, drug use for CVD, CKD, diabetic macrovascular or microvascular complications, history of severe hypoglycemia, cancer, and frailty) to minimize the confounding factors. Secondly, outcome events were ascertained from NHIS claim data, and diagnoses were not adjudicated by medical records or laboratory tests. Therefore, there can be a potential misclassification of CV outcomes. However, recent Korean studies that compared diagnoses from claim databases with medical records observed overall accuracy rates of $92.0 \%$ for MI and $90.5 \%$ for ischemic stroke [43]. Lastly, our average follow-up time was relatively short to fully assess the long-term $\mathrm{CV}$ outcomes. Further studies are warranted to assess the long-term effect of teneligliptin on risk of CVD.

To the best of our knowledge, this is the first study to compare the effect of treatment with teneligliptin to that with sulfonylurea on various $\mathrm{CV}$ outcomes in patients with T2DM using nationwide real-word data from Korea. Secondly, we used sulfonylurea as a comparator drug because it is one of the most frequently used second-line oral hypoglycemic agent after metformin in most countries and has a mechanism of action of increasing insulin similar to that of DPP-4 inhibitors. Moreover, our results are consistent with those of the CAROLINA study, a randomized controlled study comparing linagliptin with glimepiride in T2DM patients [6].

In conclusion, this study demonstrated that teneligliptin use compared to sulfonylurea use was not associated with an increased risk of $\mathrm{CV}$ events such as all-cause mortality, heart failure, and atherothrombotic events. Moreover, teneligliptin was associated with a lower risk of hypoglycemia compared to sulfonylureas. This study supported the safety of teneligliptin in patients with T2DM.

\section{CONFLICTS OF INTEREST}

This study was supported by Handok Inc., Seoul, Republic of Korea. The funders had no role in the study design, data collection and analysis, decision to publish, or manuscript preparation.

\section{ACKNOWLEDGMENTS}

This study was supported by Inha University research grant. This study used NHIS data (NHIS-2019-1-038) of the NHIS.

\section{AUTHOR CONTRIBUTIONS}

Conception or design: D.H.S., K.H.H., S.H.K., D.J.K. Acquisition, analysis, or interpretation of data: D.H.S., K.H.H., S.H.K., D.J.K. Drafting the work or revising: D.H.S., K.H.H., S.H.K., D.J.K. Final approval of the manuscript: D.H.S., K.H.H., S.H.K., D.J.K.

\section{ORCID}

Da Hea Seo https://orcid.org/0000-0003-2767-0293

Kyoung Hwa Ha https://orcid.org/0000-0002-3408-7568

So Hun Kim https://orcid.org/0000-0002-2554-3664

Dae Jung Kim https://orcid.org/0000-0003-1025-2044

\section{REFERENCES}

1. Ahren B, Foley JE. Improved glucose regulation in type 2 
diabetic patients with DPP-4 inhibitors: focus on alpha and beta cell function and lipid metabolism. Diabetologia 2016; 59:907-17.

2. Alfayez OM, Almutairi AR, Aldosari A, Al Yami MS. Update on cardiovascular safety of incretin-based therapy in adults with type 2 diabetes mellitus: a meta-analysis of cardiovascular outcome trials. Can J Diabetes 2019;43:538-45.

3. White WB, Cannon CP, Heller SR, Nissen SE, Bergenstal RM, Bakris GL, et al. Alogliptin after acute coronary syndrome in patients with type 2 diabetes. N Engl J Med 2013; 369:1327-35.

4. Scirica BM, Bhatt DL, Braunwald E, Steg PG, Davidson J, Hirshberg B, et al. Saxagliptin and cardiovascular outcomes in patients with type 2 diabetes mellitus. N Engl J Med 2013;369: 1317-26.

5. Green JB, Bethel MA, Armstrong PW, Buse JB, Engel SS, Garg J, et al. Effect of sitagliptin on cardiovascular outcomes in type 2 diabetes. N Engl J Med 2015;373:232-42.

6. Rosenstock J, Kahn SE, Johansen OE, Zinman B, Espeland MA, Woerle HJ, et al. Effect of linagliptin vs glimepiride on major adverse cardiovascular outcomes in patients with type 2 diabetes: the CAROLINA randomized clinical trial. JAMA 2019;322:1155-66.

7. Rosenstock J, Perkovic V, Johansen OE, Cooper ME, Kahn SE, Marx N, et al. Effect of linagliptin vs placebo on major cardiovascular events in adults with type 2 diabetes and high cardiovascular and renal risk: the CARMELINA randomized clinical trial. JAMA 2019;321:69-79.

8. Zannad F, Cannon CP, Cushman WC, Bakris GL, Menon V, Perez AT, et al. Heart failure and mortality outcomes in patients with type 2 diabetes taking alogliptin versus placebo in EXAMINE: a multicentre, randomised, double-blind trial. Lancet 2015;385:2067-76.

9. Liu D, Jin B, Chen W, Yun P. Dipeptidyl peptidase 4 (DPP-4) inhibitors and cardiovascular outcomes in patients with type 2 diabetes mellitus (T2DM): a systematic review and metaanalysis. BMC Pharmacol Toxicol 2019;20:15.

10. Kim KJ, Choi J, Lee J, Bae JH, An JH, Kim HY, et al. Dipeptidyl peptidase-4 inhibitor compared with sulfonylurea in combination with metformin: cardiovascular and renal outcomes in a propensity-matched cohort study. Cardiovasc Diabetol 2019;18:28.

11. Ha KH, Kim B, Choi H, Kim DJ, Kim HC. Cardiovascular events associated with second-line anti-diabetes treatments: analysis of real-world Korean data. Diabet Med 2017;34: 1235-43.
12. Lee SJ, Ha KH, Lee JH, Lee H, Kim DJ, Kim HC. Secondline glucose-lowering drugs added to metformin and the risk of hospitalization for heart failure: a nationwide cohort study. PLoS One 2019;14:e0211959.

13. Kim YG, Yoon D, Park S, Han SJ, Kim DJ, Lee KW, et al. Dipeptidyl peptidase-4 inhibitors and risk of heart failure in patients with type 2 diabetes mellitus: a population-based cohort study. Circ Heart Fail 2017;10:e003957.

14. Chin HJ, Nam JH, Lee EK, Shin JY. Comparative safety for cardiovascular outcomes of DPP-4 inhibitors versus glimepiride in patients with type 2 diabetes: a retrospective cohort study. Medicine (Baltimore) 2017;96:e7213.

15. Ha KH, Kim B, Shin HS, Lee J, Choi H, Kim HC, et al. Comparative cardiovascular risks of dipeptidyl peptidase-4 inhibitors: analyses of real-world data in Korea. Korean Circ J 2018;48:395-405.

16. Deacon CF. Dipeptidyl peptidase-4 inhibitors in the treatment of type 2 diabetes: a comparative review. Diabetes Obes Metab 2011;13:7-18.

17. Singh AK. Efficacy and safety of teneligliptin. Indian J Endocrinol Metab 2017;21:11-7.

18. Noh J. The diabetes epidemic in Korea. Endocrinol Metab (Seoul) 2016;31:349-53.

19. Seong SC, Kim YY, Khang YH, Park JH, Kang HJ, Lee H, et al. Data resource profile: the National Health Information Database of the National Health Insurance Service in South Korea. Int J Epidemiol 2017;46:799-800.

20. Austin PC. An introduction to propensity score methods for reducing the effects of confounding in observational studies. Multivariate Behav Res 2011;46:399-424.

21. Normand ST, Landrum MB, Guadagnoli E, Ayanian JZ, Ryan TJ, Cleary PD, et al. Validating recommendations for coronary angiography following acute myocardial infarction in the elderly: a matched analysis using propensity scores. J Clin Epidemiol 2001;54:387-98.

22. Rizzo MR, Barbieri M, Marfella R, Paolisso G. Reduction of oxidative stress and inflammation by blunting daily acute glucose fluctuations in patients with type 2 diabetes: role of dipeptidyl peptidase-IV inhibition. Diabetes Care 2012;35: 2076-82.

23. Matsubara J, Sugiyama S, Akiyama E, Iwashita S, Kurokawa H, Ohba K, et al. Dipeptidyl peptidase-4 inhibitor, sitagliptin, improves endothelial dysfunction in association with its anti-inflammatory effects in patients with coronary artery disease and uncontrolled diabetes. Circ J 2013;77:1337-44.

24. Ou HT, Chang KC, Li CY, Wu JS. Comparative cardiovas- 
cular risks of dipeptidyl peptidase 4 inhibitors with other second- and third-line antidiabetic drugs in patients with type 2 diabetes. Br J Clin Pharmacol 2017;83:1556-70.

25. Yu OH, Yin H, Azoulay L. The combination of DPP-4 inhibitors versus sulfonylureas with metformin after failure of first-line treatment in the risk for major cardiovascular events and death. Can J Diabetes 2015;39:383-9.

26. Kannan S, Pantalone KM, Matsuda S, Wells BJ, Karafa M, Zimmerman RS. Risk of overall mortality and cardiovascular events in patients with type 2 diabetes on dual drug therapy including metformin: a large database study from the Cleveland Clinic. J Diabetes 2016;8:279-85.

27. Scirica BM, Braunwald E, Raz I, Cavender MA, Morrow DA, Jarolim P, et al. Heart failure, saxagliptin, and diabetes mellitus: observations from the SAVOR-TIMI 53 randomized trial. Circulation 2014;130:1579-88.

28. Hashikata T, Yamaoka-Tojo M, Kakizaki R, Nemoto T, Fujiyoshi K, Namba S, et al. Teneligliptin improves left ventricular diastolic function and endothelial function in patients with diabetes. Heart Vessels 2016;31:1303-10.

29. Kosiborod M, Cavender MA, Fu AZ, Wilding JP, Khunti K, Holl RW, et al. Lower risk of heart failure and death in patients initiated on sodium-glucose cotransporter-2 inhibitors versus other glucose-lowering drugs: the CVD-REAL study (comparative effectiveness of cardiovascular outcomes in new users of sodium-glucose cotransporter-2 inhibitors). Circulation 2017;136:249-59.

30. Kim YG, Han SJ, Kim DJ, Lee KW, Kim HJ. Association between sodium glucose co-transporter 2 inhibitors and a reduced risk of heart failure in patients with type 2 diabetes mellitus: a real-world nationwide population-based cohort study. Cardiovasc Diabetol 2018;17:91.

31. Kirkman MS, Briscoe VJ, Clark N, Florez H, Haas LB, Halter JB, et al. Diabetes in older adults. Diabetes Care 2012; 35:2650-64.

32. Go AS, Mozaffarian D, Roger VL, Benjamin EJ, Berry JD, Borden WB, et al. Executive summary: heart disease and stroke statistics. 2013 Update: a report from the American Heart Association. Circulation 2013;127:143-52.

33. Bleumink GS, Knetsch AM, Sturkenboom MC, Straus SM, Hofman A, Deckers JW, et al. Quantifying the heart failure epidemic: prevalence, incidence rate, lifetime risk and prog- nosis of heart failure The Rotterdam Study. Eur Heart J 2004;25:1614-9.

34. Alonso-Moran E, Orueta JF, Fraile Esteban JI, Arteagoitia Axpe JM, Marques Gonzalez ML, Toro Polanco N, et al. The prevalence of diabetes-related complications and multimorbidity in the population with type 2 diabetes mellitus in the Basque Country. BMC Public Health 2014;14:1059.

35. American Diabetes Association. 9. Pharmacologic approaches to glycemic treatment: Standards of Medical Care in Diabetes-2019. Diabetes Care 2019;42(Suppl 1):S90-102.

36. Kim MK, Ko SH, Kim BY, Kang ES, Noh J, Kim SK, et al. 2019 Clinical practice guidelines for type 2 diabetes mellitus in Korea. Diabetes Metab J 2019;43:398-406.

37. Aschner P, Kipnes MS, Lunceford JK, Sanchez M, Mickel C, Williams-Herman DE, et al. Effect of the dipeptidyl peptidase-4 inhibitor sitagliptin as monotherapy on glycemic control in patients with type 2 diabetes. Diabetes Care 2006; 29:2632-7.

38. Pi-Sunyer FX, Schweizer A, Mills D, Dejager S. Efficacy and tolerability of vildagliptin monotherapy in drug-naïve patients with type 2 diabetes. Diabetes Res Clin Pract 2007; 76:132-8.

39. Rosenstock J, Aguilar-Salinas C, Klein E, Nepal S, List J, Chen $\mathrm{R}$, et al. Effect of saxagliptin monotherapy in treatment-naïve patients with type 2 diabetes. Curr Med Res Opin 2009;25:2401-11.

40. Kagansky N, Levy S, Rimon E, Cojocaru L, Fridman A, Ozer Z, et al. Hypoglycemia as a predictor of mortality in hospitalized elderly patients. Arch Intern Med 2003;163: 1825-9.

41. Leiter LA, Teoh H, Braunwald E, Mosenzon O, Cahn A, Kumar KM, et al. Efficacy and safety of saxagliptin in older participants in the SAVOR-TIMI 53 trial. Diabetes Care 2015;38:1145-53.

42. Abubaker M, Mishra P, Swami OC. Teneligliptin in management of diabetic kidney disease: a review of place in therapy. J Clin Diagn Res 2017;11:OE05-9.

43. Park J, Kwon S, Choi EK, Choi Y, Lee E, Choe W, et al. Validation of diagnostic codes of major clinical outcomes in a National Health Insurance database. Int J Arrhythm 2019; $20: 5$. 